\title{
L'antiquaire Georges Joseph Demotte, le Louvre et les musées américains. S'approprier le discours sur le patrimoine médiéval de la France au sortir de la Première Guerre mondiale
}

The antiquarian Georges Joseph Demotte, the Louvre and American museums: appropriating the discourse on the medieval heritage of France at the end of the First World War

\section{Christine Vivet-Peclet}

\section{(2) OpenEdition}

\section{Édition électronique}

URL : http://journals.openedition.org/cel/705

DOI : $10.4000 / \mathrm{cel} .705$

ISSN : 2262-208X

Éditeur

École du Louvre

\section{Référence électronique}

Christine Vivet-Peclet, « L'antiquaire Georges Joseph Demotte, le Louvre et les musées américains. S'approprier le discours sur le patrimoine médiéval de la France au sortir de la Première Guerre mondiale », Les Cahiers de l'École du Louvre [En ligne], 11 | 2017, mis en ligne le 26 octobre 2017, consulté le 17 septembre 2019. URL : http://journals.openedition.org/cel/705; DOI : 10.4000/cel.705

Ce document a été généré automatiquement le 17 septembre 2019.

Les Cahiers de l'École du Louvre sont mis à disposition selon les termes de la licence Creative Commons Attribution - Pas d'Utilisation Commerciale - Pas de Modification 4.0 International. 


\section{L'antiquaire Georges Joseph} Demotte, le Louvre et les musées américains. S'approprier le discours sur le patrimoine médiéval de la France au sortir de la Première Guerre mondiale

The antiquarian Georges Joseph Demotte, the Louvre and American museums: appropriating the discourse on the medieval heritage of France at the end of the First World War

\section{Christine Vivet-Peclet}

1 L'antiquaire Georges Joseph Demotte (1877-1923) a joué un rôle important pour la diffusion et la connaissance de l'art médiéval en France et aux États-Unis dans les années de l'immédiat après-guerre. À Paris, il est présent au musée du Louvre, à la fois grâce aux œuvres qui sont acquises auprès de lui ou par son intermédiaire mais aussi en tant qu'éditeur. Aux États-Unis, il propose aux grands collectionneurs et aux musées des objets qui vont contribuer à la découverte puis à l'étude de l'art médiéval français ${ }^{1}$.

\section{La promotion de l'art médiéval}

2 Avec son fils Lucien, Georges Demotte a été l'un des acteurs essentiels du marché de l'art du premier tiers du $\mathrm{xx}^{\mathrm{e}}$ siècle. Le travail qui est actuellement effectué sur le fonds photographique conservé au département des Sculptures du musée du Louvre ${ }^{2}$ permet d'appréhender leur importance dans les domaines dont ils s'étaient fait la spécialité, à travers l'étude des œuvres reproduites. Elles dessinent l'activité de Georges Joseph 
Demotte (1877-1923), puis de son fils Lucien Demotte (1906-1934) qui lui succède à la tête de l'entreprise familiale après son décès accidentel ${ }^{3}$.

La période qui va de 1913 au début des années 1920 est un moment clé dans l'activité de Demotte père. À Paris, il quitte en 1913 sa boutique de la rue de Provence et le quartier $\mathrm{du} 9^{\mathrm{e}}$ arrondissement, où sont installés un bon nombre de ses collègues, dont Charles Vignier $^{4}$, rue Laffitte, qui, comme lui, consacre une partie de son activité à la vente d'objets d'art persans. Il rejoint le quartier plus prestigieux des Champs-Élysées et s'installe dans un hôtel particulier, malheureusement disparu, 27 rue de Berri (fig. 1). Il $\mathrm{y}$ organise des expositions où sont présentés dans une mise en scène très théâtrale œuvres et éléments d'architecture médiévaux. L'exposition inaugurale est relayée par la presse qui relève l'originalité et la nouveauté d'une telle proposition présentant un choix important d'œuvres gothiques ${ }^{5}$. Avec cette nouvelle installation il se positionne comme le spécialiste incontesté dans ce domaine et son activité prospère. En témoigne un courrier du 15 septembre 1916 qu'il adresse à son collègue et ami George Grey Barnard (1863-1938) ${ }^{6}$. Il y mentionne qu'il a gagné de juillet 1913 à juillet 1914 la somme de $600000 \$$ soit 3000000 francs $^{7}$, somme assez considérable pour l'époque.

Fig. 1

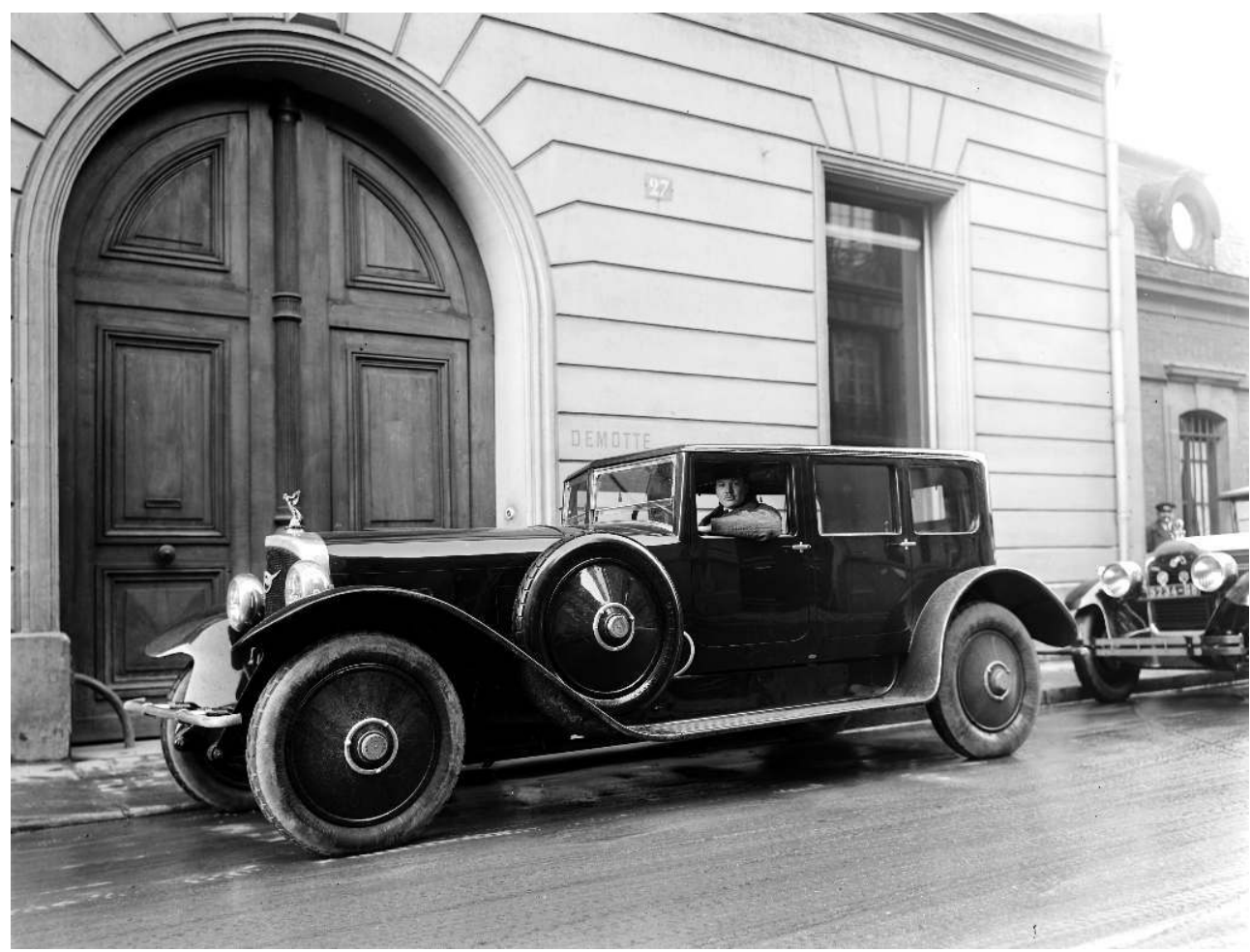

La galerie Demotte à Paris, 27 rue de Berry

(C) Paris, musée du Louvre, département des Sculptures, fonds Demotte

Georges Demotte ouvre une succursale de sa galerie à New York en avril 1918 au 8 East 57th Street ${ }^{8}$. Il n'est pas le premier des antiquaires parisiens à le faire, mais il se démarque, tout comme à Paris, par la singularité de ses choix en proposant presque exclusivement des œuvres dites gothiques, présentées dans un immeuble entièrement réaménagé à cet effet (fig. 2). La presse américaine accompagne l'ouverture de cette galerie en lui consacrant de nombreux articles où sont décrites les œuvres proposées 9 . 
Tous soulignent que l'ouverture d'une telle galerie est opportune dans un moment où les Américains éprouvent de la sympathie pour la France, victime de la guerre et de destructions de son patrimoine.

Fig. 2

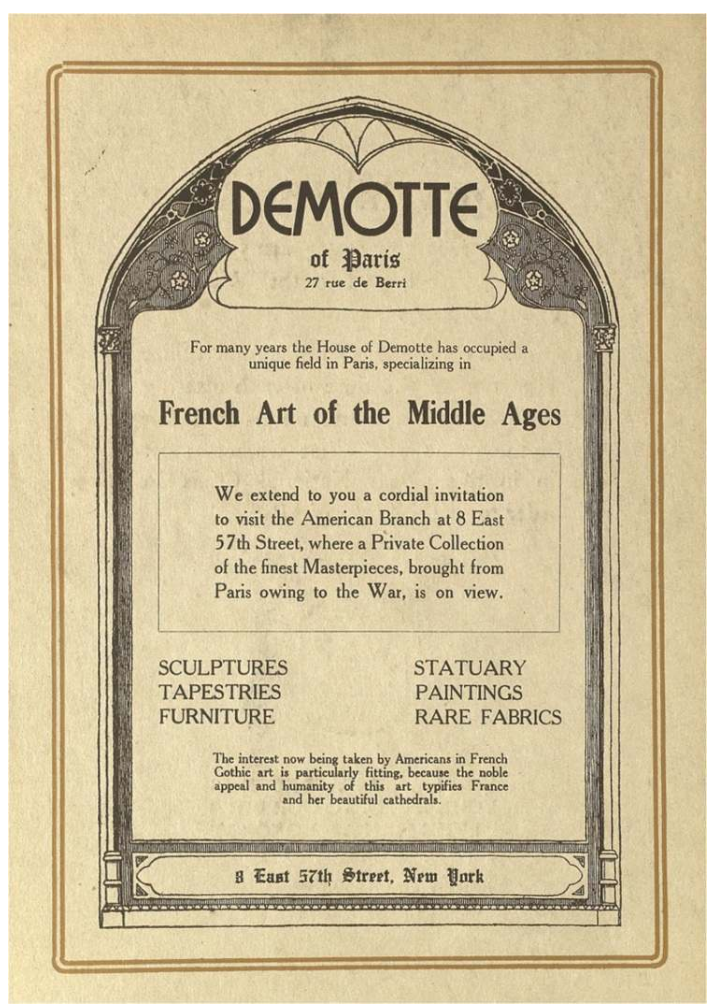

Encart publicitaire annonçant l'ouverture de la galerie Demotte à New York, d'après Fine Arts Journal, vol. $36, n^{\circ} 10$, octobre 1918

\section{La vie au Louvre durant la Première Guerre mondiale}

Durant tout le conflit, si les collections de peintures ont été déménagées vers Toulouse, les sculptures médiévales et modernes sont restées sur place, laissées à leur emplacement d'origine dans les salles du rez-de-chaussée de la Cour carrée. Ces salles sont réouvertes provisoirement au public du mois de mars 1916 au 7 février 1917, puis du $1^{\text {er }}$ mai 1917 au 31 janvier $1918^{10}$. C'est ainsi que des œuvres moins connues et moins prisées en général par le public habituel du musée sont les seules visibles. Les articles qui paraissent alors dans la presse relèvent cette spécificité décrite par André Michel (1843-1925), conservateur au département des Sculptures, dans le Journal des débats politiques et littéraires ${ }^{11}$ :

Il n'est peut-être pas indifférent, à ce point de vue, de remarquer que, par suite de circonstances d'ailleurs toutes fortuites, c'est la section la plus française du musée qui aura l'honneur d'accueillir la première la visite des Parisiens.

Y sont présentées les acquisitions récentes et en particulier trois œuvres romanes provenant de Notre-Dame-de-la-Couldre à Parthenay ${ }^{12}$, acquises auprès de Demotte et qui sont le plus citées et commentées dans la presse ${ }^{13}$. Demotte peut ainsi écrire à son ami Barnard : 
The Louvre has just opened a room for my XIIth century statue (bas reliefs) specially for them and they met with the greatest interest from the public. The only rooms open at the Louvre are the gothic statues rooms - this will give you an idea of the mentality of people here concerning gothic and the interest collectors take in that art - even people who until now - had only been buyers of XVIIIth century paintings sculptures or meubles ${ }^{14}$.

Malgré la guerre, le musée poursuit l'accroissement de ses collections grâce à de nombreux dons et legs mais aussi par quelques acquisitions importantes. Parmi cellesci, on peut noter l'achat d'un tableau de Louis Le Nain ${ }^{15}$, acquis auprès de Demotte après qu'il en a fait l'acquisition lors d'une vente aux enchères où le Louvre, présent, avait renoncé. De grands collectionneurs, clients habituels de Demotte, font des dons importants à la section des arts de l'Islam et en particulier Joanny Peytel (1844-1924), financier, et Georges Marteau (1858-1916), maître-cartier. Le département des Sculptures reçoit également un don de plusieurs sculptures de la collection FerdinandEdmond Jeuniette (1854-1918), parmi lesquelles se trouvent des œuvres vendues à ce dernier par Demotte. Ainsi, ce dernier fournit directement ou indirectement des pièces maitresses venant enrichir les collections du musée du Louvre.

\section{Un éditeur au service du musée}

7 Au sortir de la guerre, il est décidé de présenter au public les œuvres acquises par le musée durant cette période. On procède à l'aménagement provisoire de la salle La Caze ${ }^{16}$ et cette manifestation fait tout d'abord l'objet d'une publication de la maison Braun sous forme d'un guide bilingue de 173 pages, dont 16 pages de reproductions ${ }^{17}$. Demotte va proposer au Louvre de publier un ouvrage qui présente une sélection de ces œuvres. Après avoir obtenu l'autorisation du musée ${ }^{18}$, il s'associe à Henri Rivière (1864-1951) ${ }^{19}$ qui sera le directeur artistique de ce projet. Le livre parait en 1920 en deux volumes (fig. 3). L'introduction est signée par Louis Barthou (1862-1934) ${ }^{20}$. Reprise telle quelle dans certains articles de presse ${ }^{21}$, elle salue l'action de "salut public » menée par Demotte :

Ce livre est un témoin. Il parle pour la France, dont il atteste, une fois de plus et sous une forme nouvelle, le courage clairvoyant et la tranquille confiance pendant les quatre années tragiques où la guerre menaça son existence. Tandis que les soldats se battaient aux frontières, rapprochées hélas ! sur le sol même de la Patrie que souillait l'envahisseur, l'arrière faisait son devoir. [...] Le Musée du Louvre ne fut inférieur ni à sa tâche ni à sa gloire. Lui aussi, il tint. [...] M. Demotte, habitué par sa profession au contact des objets d'art, à leur goût et à leur conservation, a pris une initiative dont l'audace mérite d'être heureuse. Il a consacré aux acquisitions du Louvre pendant la guerre un livre magnifique qui restera comme le monument superbe d'une activité jusqu'ici mal connue et digne pourtant de tous les hommages. Cette œuvre, née d'une pensée juste et généreuse, est le témoin du Louvre, sauvé et enrichi.

C'est une édition luxueuse, composée de cent grandes planches en deux tomes avec des reproductions des œuvres en pleine page, en noir et blanc ou en couleur et accompagnées de notices détaillées signées par les conservateurs du musée. Une table des matières à la fin de chaque volume renvoie aux titres des œuvres, aux artistes et aux auteurs des notices. À l'intérieur des deux tomes le classement des œuvres se fait par catégories d'objets sans distinction de périodes et d'écoles. Le premier volume est consacré aux Sculptures/Peintures, le second aux Dessins/Objets d'art. L'ouvrage ne présente aucune des œuvres acquises directement auprès de Demotte dans les 
départements modernes, exception faite du tableau de Louis Le Nain ${ }^{22}$. En revanche, sont présentées en majorité les œuvres entrées dans les collections grâce aux dons faits par ses principaux clients, comme l'ange en bois, don Jeuniette ${ }^{23}$, ou les œuvres données à la section islamique par Peytel et Marteau. La presse salue la qualité et la nécessité de cet ouvrage :

Ce magnifique ouvrage, impatiemment attendu depuis si longtemps, est accueilli avec une faveur unanime. Il réjouit et console à la fois. C'est, en effet, avec une joie infinie que l'amateur de livres le plus difficile à satisfaire en analyse tous les détails de fabrication et que l'amateur d'art le mieux renseigné s'attarde dans l'examen d'une présentation générale où tout a été l'objet du soin le plus attentif et le plus clairvoyant. Et puis, il faut bien le dire, notre sentiment national est à la fois consolé et rassuré par l'apparition de ce beau spécimen de notre librairie d'art qui, depuis des années, ne brillait pas de l'éclat le plus pur au ciel de l'édition internationale ${ }^{24}$.

On salue également le fait que la publication permette de présenter au public la vie du musée sur le modèle des bulletins qui existent dans d'autres musées européens ou américains $^{25}$ :

En attendant que le directeur actuel des Musées nationaux puisse réaliser le projet qu'on lui prête de la ressusciter, voici qu'un simple éditeur (doublé, il est vrai, d'un « antiquaire " considérable) nous donne, par ses moyens privés, ce magnifique album où se reflète toute l'existence du Louvre pendant les cinq années de guerre 1914-1919.

Toutefois, quelques voix s'élèvent pour noter qu'il reste inabordable pour la majorité du public ${ }^{26}$ :

Aussi devons-nous témoigner une particulière reconnaissance à l'Antiquaire considérable et érudit qu'est M. Demotte qui, pour la meilleure mise en valeur de nos nouvelles richesses artistiques, s'est improvisé éditeur d'art et vient de nous faire connaître, dans une somptueuse publication, les accroissements du Musée du Louvre depuis 1914 [...]. On aime, avant d'aller les voir à leur place nouvelle, à feuilleter les belles héliogravures qui nous les présentent [...]. Il faut s'arrêter cependant, mais non toutefois sans exprimer le désir que ces deux magnifiques volumes, vraiment inaccessibles et par leur prix et leur format au grand public, soient résumés en une plaquette très condensée, illustrée de simples gravures au trait, avec l'indication de la place où se trouvent les petits monuments nouveaux, de façon que les visiteurs de notre admirable Musée puissent les examiner à loisir et apprécier l'intérêt des nouvelles acquisitions, dans les lacunes qu'elles sont venues combler.

De fait, cette publication ne connaitra pas de postérité, sans doute car trop luxueuse. Alors qu'il était initialement prévu de publier annuellement les acquisitions du musée, seul un premier volume consacré aux acquisitions de 1920 parait en $1921^{27}$. 
Fig. 3

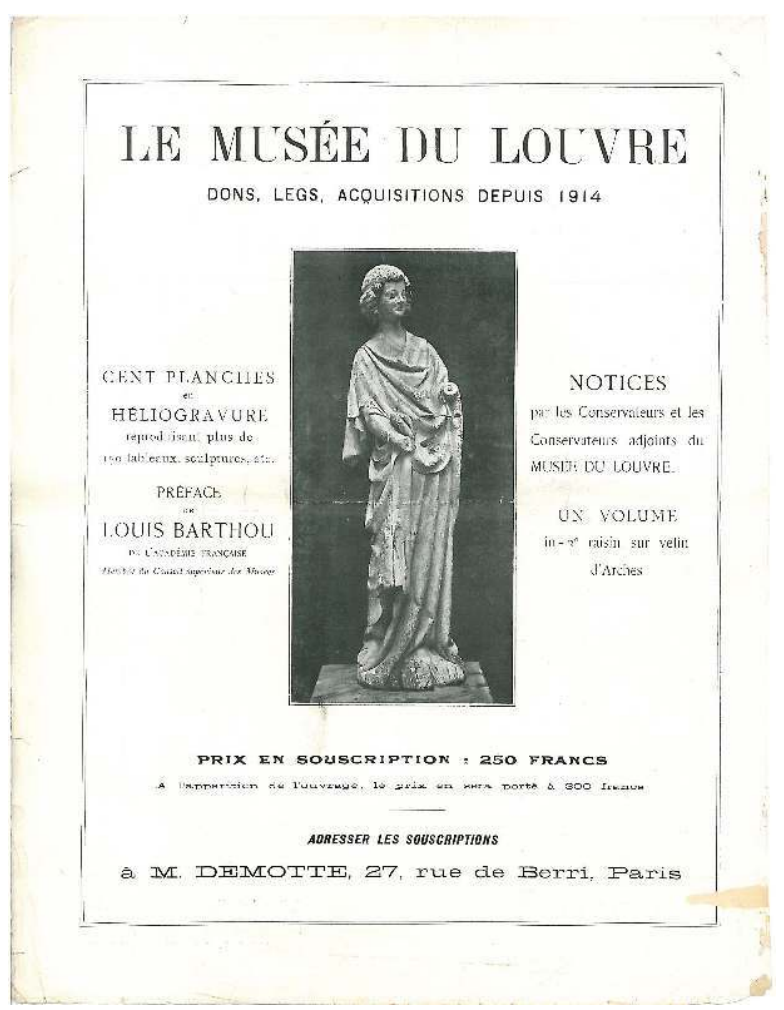

Bon de souscription pour l'ouvrage sur les acquisitions du Louvre durant la guerre, d'après Les Arts, 1919

\section{Une appropriation de l'image du musée du Louvre}

Les motivations de Demotte sont multiples lorsqu'il se lance dans une telle entreprise. Il y a chez lui la volonté et l'ambition de publier des ouvrages d'art de référence. En effet, à la suite de cette première publication, il édite en 1922 un ouvrage sur les dessins de Degas ${ }^{28}$ (qu'il envisage comme le premier d'une série sur les dessins de peintres français) et, la même année, un ouvrage en deux volumes sur le sculpteur Falconet signé de Louis Réau (1881-1961) ${ }^{29}$. Il commence aussi à publier un ambitieux ouvrage sur la tapisserie gothique entre 1921 et $1924^{30}$ et avait pour dessein de publier une encyclopédie de l'art du Portugal. Sa mort accidentelle mettra fin à ses différents projets, que son fils Lucien décidera de ne pas poursuivre. La qualité des ouvrages publiés impliquait un coût important de fabrication. Lucien, quelques années plus tard, parlant de l'activité d'éditeur de son père, note d'ailleurs qu'elle n'a jamais été rentable $^{31}$. Si la vente directe de tels ouvrages ne permet pas de faire de profit, Demotte les utilise comme objet de promotion. Il n'hésite pas à les adresser en hommage à ses plus prestigieux clients. Par exemple, il en envoie un exemplaire à Raymond Pitcairn (1885-1966) en $1921^{32}$. Dans les courriers qui accompagnent cet envoi, il liste les vingttrois œuvres acquises auprès de lui parmi les cent que compte l'ouvrage. Il semble avoir répété cette opération auprès de quelques-uns de ses clients américains, collectionneurs ou musées. Ainsi, le bulletin publié par le musée de Saint Louis mentionne le don fait en avril 1922 par Demotte des trois volumes de ce même 
ouvrage ${ }^{33}$. Demotte s'approprie ainsi l'aura du musée du Louvre et grâce à cela peut légitimement se présenter comme «fournisseur » attitré de celui-ci.

\section{Les collections d'art médiéval aux États-Unis}

De fait l'antiquaire, avant même son installation définitive à New York, a su s'implanter sur le marché américain et compte parmi ses clients de très grands collectionneurs. Grâce à une politique commerciale active et astucieuse alternant l'envoi d'ouvrages, l'envoi de photographies, l'organisation d'expositions temporaires dans ses galeries, et des dons réguliers à de nombreuses institutions, il s'établit durablement sur le sol américain. Ainsi sur les 921 œuvres «Demotte » actuellement localisées grâce aux photographies présentes dans la base, 607 sont conservées dans des collections américaines publiques ou privées. Selon les institutions, il s'agit d'une œuvre isolée ou d'ensembles plus importants, qui peuvent compter plus d'une centaine d'œuvres.

Jusqu'à la fin du xixe siècle c'est d'abord l'aspect décoratif de l'art médiéval qui est recherché par les décorateurs professionnels afin de créer pour leurs clients des Period Rooms ou d'agrémenter leurs intérieurs en créant dans leurs résidences une pièce dite gothique. Cette démarche s'inscrit dans la poursuite du modèle proposé en France par le musée des Monuments français d'Alexandre Lenoir ${ }^{34}$ (qui a fermé ses portes en 1816), et dont on trouve un écho au musée de Cluny qui ouvre en 1843 et où les œuvres sont mises en scène dans un décor. Le musée de Sculpture comparée qui ouvre ses portes en 1882 présente quant à lui des moulages des monuments les plus importants de l'art français classés de manière chronologique et propose un discours d'histoire de l'art. Il aura également une grande influence sur les collections américaines et, avant la constitution des premières collections d'œuvres originales, c'est d'ailleurs par des moulages que l'art médiéval est surtout connu aux États-Unis. Puis, au début du $\mathrm{xx}^{\mathrm{e}}$ siècle, grâce aux premiers grands collectionneurs que sont Isabella Stewart Gardner à Boston (1840-1924), John Pierpont Morgan (1837-1913) à New York, Henry Walters (1848-1931) à Baltimore et Mary Mitchell Blair (1866-1943) à Chicago, arrivent sur le sol américain les premières œuvres médiévales originales. Il s'agit souvent de quelques objets s'intégrant dans une collection plus importante d'œuvres françaises de périodes et de techniques différentes. Leur présentation dans les musées qu'ils vont fonder ou honorer de leurs dons est alors très proche de celle de leurs propres intérieurs domestiques.

\section{Un musée entièrement consacré à l'art médiéval}

11 Quelques années plus tard, il revient à Georges Grey Barnard de proposer au public américain le premier musée entièrement consacré à l'art médiéval. Lors de son séjour en France il achète, chez des antiquaires et des particuliers, des sculptures et fragments architecturaux, en particulier des ensembles de cloîtres entiers ${ }^{35}$ tant pour lui-même que pour les vendre à d'autres amateurs. À son retour aux États-Unis, en 1915, il va présenter sa collection au public à New York dans un bâtiment en briques sur Fort Washington Avenue (fig. 4). 
Fig. 4

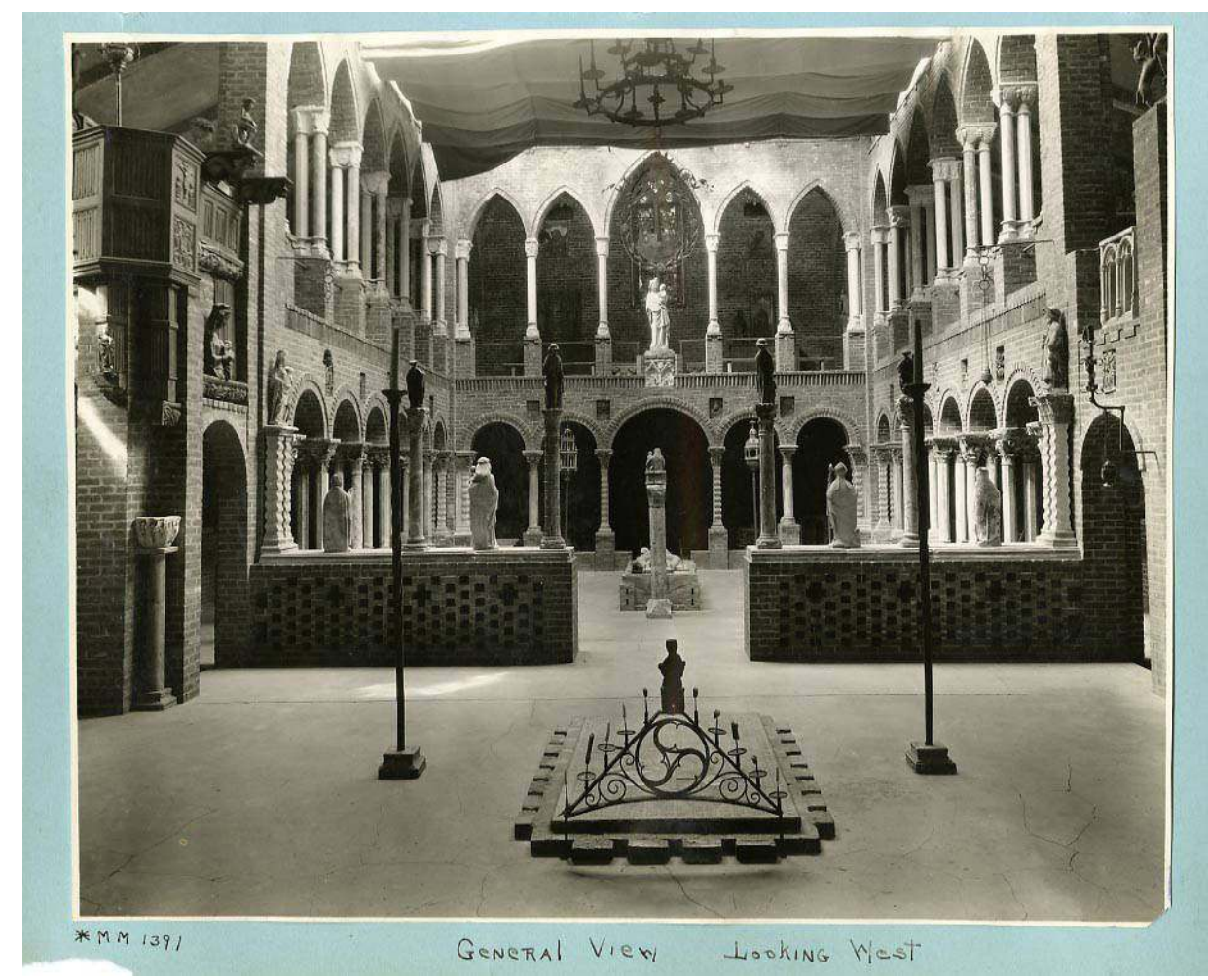

Vue générale de l'intérieur des premiers cloitres de George Grey Barnard, Views of the Cloisters, New York, Metropolitan Museum of Art, The Cloisters Archives

Barnard et Demotte se connaissent et se fréquentent. La correspondance ${ }^{36}$ qui s'établit entre eux montre qu'en dehors de relations purement professionnelles, il existe une solide amitié entre les deux hommes, qui ont dû se rencontrer au moment où Barnard vivait en France. Barnard conserve son amitié à Demotte y compris dans les moments où celui-ci est au cœur de scandales ${ }^{37}$, Barnard étant alors l'une des seules personnes à prendre publiquement sa défense. Il fut aussi le premier intermédiaire de Demotte aux États Unis et l'encouragea à s'y installer. Demotte aida Barnard à rapatrier les dernières œuvres qu'il avait laissées en France, ce qui n'alla pas sans difficultés. En effet, Barnard quitta la France au moment où l'État français commençait à prendre des mesures pour protéger son patrimoine ${ }^{38}$ (mesures dictées pour une bonne part par les agissements de Barnard lui-même et l'indignation que son action déclencha auprès du peuple français). On connaît les motifs qui poussèrent Barnard à ouvrir "son musée " grâce aux guides et aux articles parus dans la presse ${ }^{39}$. Le premier de ses buts est de donner des modèles pour la taille de la pierre à ses étudiants sculpteurs. Le second est de replacer les œuvres dans leur contexte afin que les visiteurs puissent voir quelque chose du passé et s'imprégner des sentiments que les œuvres inspirent sans aller jusqu'en Europe. Pour cela, il organise dans le musée une véritable mise en scène. Les guides accompagnant les visiteurs sont déguisés en moines, une musique médiévale d'ambiance est diffusée et l'éclairage des salles se fait aux chandelles. Des revers de fortune vont contraindre Barnard à vendre ce musée. Après de nombreuses tractations c'est John D. Rockefeller Junior (1874-1960) ${ }^{40}$ qui s'en porte acquéreur et le donne au Metropolitan Museum of Art pour en faire une annexe. Ouvert en 1925, il sera ensuite fermé puis reconstruit sur un autre site et ouvert au public en 1938 à l'endroit qu'il occupe encore aujourd'hui, à Fort Tryon. Un article bilingue de Jean Vallery-Radot (1890-1971), intitulé « Pierres de 
France en Amérique ${ }^{41}$ ", paru à l'occasion de l'ouverture des Cloisters de 1925 est représentatif de l'attitude de la France face à un tel projet. Il commence par déplorer que l'Europe ne soit pas assez protégée par ses lois contre l'évasion des œuvres d'art. «On assiste au $\mathrm{xx}^{\mathrm{e}}$ siècle à un transfert du goût qui attire non plus vers les Antiques mais vers les vestiges d'architecture médiévale que le marteau de la Révolution a disséminés en France ». Une seconde partie de l'article est consacrée à la description du musée. Il note l'effort fait par Barnard qui tente de reconstituer un cadre, en parfait accord avec les objets exposés, en construisant une véritable église avec une nef, un chœur et une sacristie mais souligne que cet agencement fait plutôt penser à un décor de théâtre ou à un atelier d'artiste qu'à un musée. Dans sa conclusion il commence par déplorer le départ et la dispersion du patrimoine national mais souligne que dans le même temps cela contribue au rayonnement international de la France :

[...] L'étrange fortune de ces œuvres d'art de notre pays émigrées sur les bords de l'Hudson River, prête matière à réflexion et ces réflexions seraient pour un Français fâché d'être ainsi appauvri dans son patrimoine artistique, d'ordre plutôt mélancolique, si l'attraction exercée par notre art national à l'étranger n'était pas, après tout, un légitime sujet d'orgueil, et si l'on ne songeait aussi au rôle éminemment éducateur que vont jouer, outre-Atlantique, ces émouvantes pierres de France [...].

\section{Un projet avorté - Un mémorial de l'art gothique}

12 À la suite de l'ouverture publique de sa propre collection et probablement en raison du succès de celle-ci, Barnard propose à Rockefeller de faire de même en créant un «mémorial de l'art gothique ${ }^{42}$ ». Pour réaliser ce projet, Barnard sollicite Demotte qui doit lui fournir une centaine d'œuvres. Selon ses recommandations, l'opération se fera dans le plus grand anonymat, Barnard servant d'intermédiaire entre le milliardaire et l'antiquaire qui ignorera l'identité de son client. Pour l'accompagner dans cette entreprise, Rockefeller fait également appel à son architecte en titre William Welles Bosworth (1869-1966) afin de construire l'édifice qui abritera cette collection ${ }^{43}$. Bien que les œuvres aient été acquises et expédiées à New York dès la fin de l'année 1916, il semble qu'un désaccord soit survenu entre Barnard et Bosworth et le projet n'aboutira finalement pas. L'organisation de ce projet est l'occasion d'échanges nombreux entre les différents protagonistes ${ }^{44}$. Les échanges entre Barnard et Demotte concernent la commande et l'envoi de photographies de 271 œuvres dont 100 seront sélectionnées. Les échanges entre Barnard et Rockefeller permettent au premier d'exposer son projet, la construction d'un mémorial en hommage à l'art gothique où seraient présentées des œuvres isolées et non des cloîtres entiers, comme dans son propre musée. Son souhait est de construire une collection avec une atmosphère aussi superbe que celle du musée de Cluny à Paris et d'en faire un lieu sacré unique par sa beauté et sa paix, pour les artistes, les poètes et toute la population. Le projet est approuvé par Bosworth qui, dans ses échanges avec Rockefeller, précise la nature de ce lieu qui doit permettre de présenter au public et pour le bien de l'humanité un ensemble d'« œuvres sacrifiées ", recueillies dans les ruines du nord de la France et de la Belgique. Après l'abandon du projet, les œuvres finiront, pour certaines, par rejoindre les collections du Metropolitan Museum of Art à New York et du Museum of Art de Cleveland et d'autres resteront entre les mains de la famille Rockefeller sur la propriété familiale où elles sont toujours conservées ${ }^{45}$. 


\section{Un discours sur l'art médiéval}

Le moment où les premiers Cloisters de Barnard ouvrent au public s'accompagne d'un discours d'appropriation du patrimoine de l'humanité en danger, qui se retrouve dans deux articles qui annoncent l'ouverture de la galerie Demotte à New York. Le premier, «Regarding the Demotte Galleries ${ }^{46}$ » est un profond plaidoyer en faveur de l'art gothique. Il rappelle le contexte de la guerre et des destructions qui ont fait prendre conscience en France et dans le monde de l'importance de ce patrimoine détruit ou menacé. Il souligne la valeur morale, la dévotion, le travail artisanal des hommes qui ont créé cet art. Il conclut avec emphase sur l'esprit de la France que représente l'art gothique et qui ne peut pas être détruit :

[...] French towns may be destroyed, French soldiers killed, but the spirit of France, old and finely wrought, is immortal: Rheims may be levelled to their ground, Amiens may perish, but the spirit of Gothic art, old and finely wrought, is immortal, and cannot by mortals be destroyed.

Le second, "By buying her gothic art world will aid France in a new way ${ }^{47}$ ", développe un tout autre discours. Les œuvres présentent en plus de leur valeur artistique un intérêt particulier au moment où ce patrimoine est en danger. Il y a la nécessité de procurer de l'aide aux habitants des régions dévastées et cela peut être fait en achetant des œuvres d'art car le commerce des antiquités fait partie des quatre branches dans lesquelles les Français peuvent continuer de faire des affaires y compris en temps de guerre. Ces deux textes exposent ainsi certains des motifs qui vont inciter les collectionneurs américains à se porter acquéreurs du patrimoine français, en particulier médiéval.

\section{Profils de collectionneurs}

Demotte va compter parmi ses prestigieux clients des collectionneurs dont les motivations se lisent à travers le choix d'œuvres dont ils se portent acquéreurs.

Isabella Stewart Gardner, philanthrope et mécène, est la première américaine à acheter des sculptures romanes françaises (fig. 5), et cela avant même l'ouverture des Cloisters de Barnard ${ }^{48}$. Elle constitue sa collection, Fenway Court, non comme un musée mais comme un palais italien de la Renaissance et rassemble les œuvres dans des pièces où elles sont mises en scène en même temps que sa propre figure de collectionneuse. Ainsi, dans la salle qu'elle construit autour de sa collection médiévale, trône son portrait en impératrice byzantine par le peintre John Singer Sargent (1856-1925). Elle sait toutefois s'entourer de l'avis d'experts qui l'encouragent et la conseillent dans ses achats, comme Bernard Berenson (1865-1959), historien de l'art américain, spécialiste de la Renaissance italienne. Sa collection, qu'elle ne cessera d'enrichir durant toute sa vie, visible quelques jours par an jusqu'à sa mort en 1924, est ensuite ouverte définitivement au public. 
Fig. 5

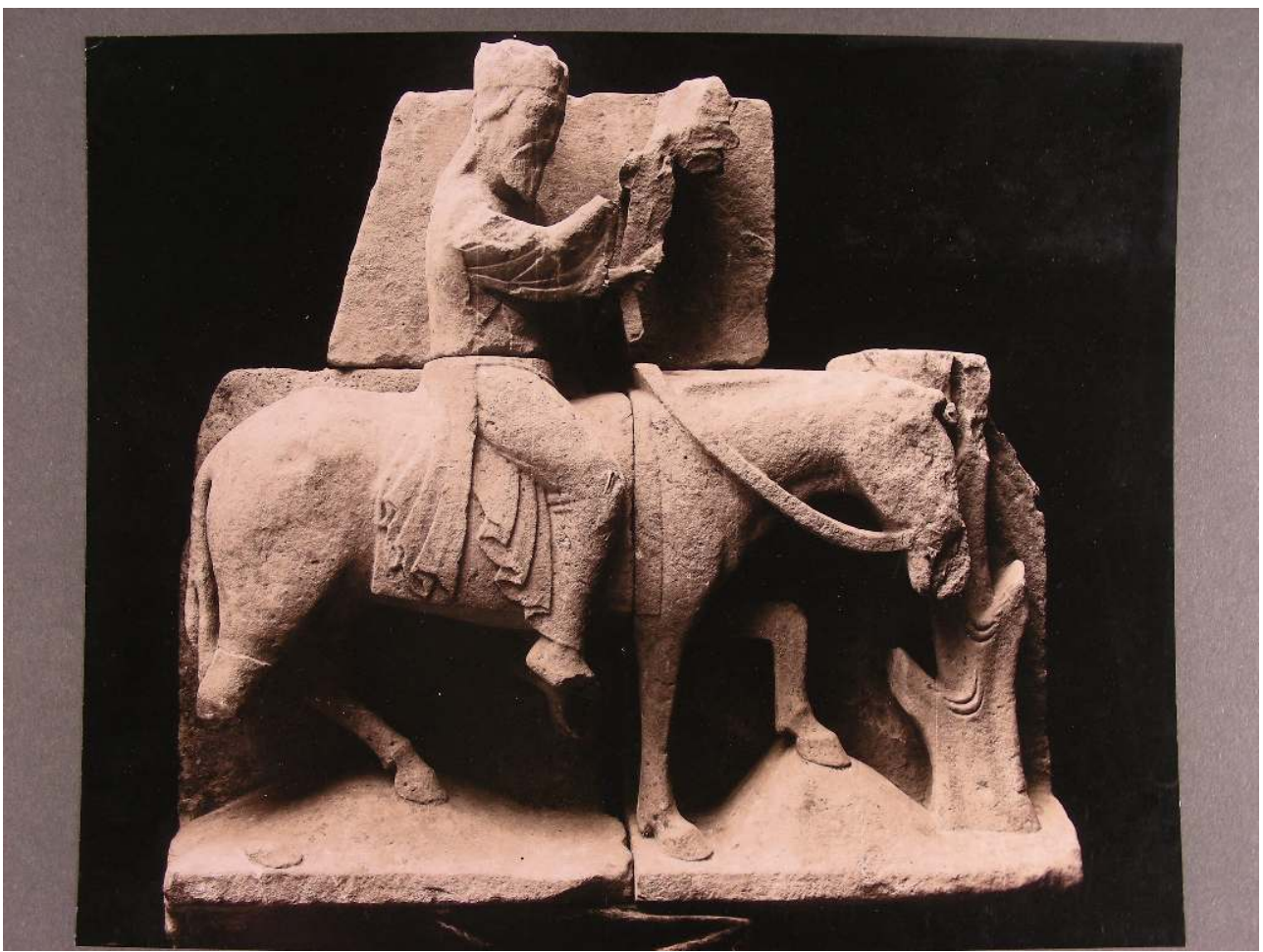

ENTRÉE DU CHRIST À JÉRUSALEM, BOSTON ISABELLA STEWART GARDNER MUSEUM (INV. S7N1), NEW YORK, METROPOLITAN MUSEUM OF ART, THE CLOISTERS ARCHIVES, DEMOTTE PHOTO ARCHIVE

Avec une ambition spirituelle et dans la tradition de la promotion de l'artisanat, Raymond Pitcairn, avocat, homme d'affaires et membre éminent de l'Église de la New Church ${ }^{49}$, rassemble une importante collection de vitraux mais également de sculptures romanes et gothiques. L'objectif premier de sa collection est de proposer des modèles aux artisans qui construisent entre 1913 et 1938 la cathédrale qu'il fait bâtir à Bryn Athyn ${ }^{50}$. L'achat d'œuvres médiévales authentiques est une préoccupation constante du collectionneur. Elles représentent un retour aux sources et sont choisies pour leur valeur spirituelle et religieuse. Les objets, qu'il négocie toujours aux prix les plus bas, sont d'abord conservés sur la propriété familiale bâtie par son père. L'ampleur de la collection, qui ne cesse de s'accroître, le décide à faire construire une maison où les objets ont chacun un emplacement spécifique. Elle restera une maison de famille jusque dans les années 1970, à la mort de l'épouse de Raymond. La famille décide alors de la donner ainsi que ses collections à l'Église qui la transforme en un musée des religions, illustrées par des œuvres authentiques de toutes les civilisations. La collection comprend une part très importante d'art médiéval (vitraux et sculptures). Une partie de la maison a été conservée telle qu'elle a été voulue par Pitcairn, c'est par exemple le cas du Great Hall. Le musée a connu des aménagements récents avec des espaces dédiés aux œuvres qui permettent de présenter les chefs-d'œuvre de la collection. En dehors des collections du Metropolitan Museum of Art, il s'agit d'une des plus importantes collections d'art médiéval conservées aux États-Unis.

16 C'est un autre profil de collectionneur que celui de l'homme d'affaires et magnat de la presse William Randolph Hearst (1863-1951). C'est un acheteur encyclopédique qui s'approprie en masse une quantité colossale d'œuvres. Il possède de multiples propriétés à la manière de châteaux où il fait aménager des salles gothiques mais aussi 
des piscines où prennent place de véritables cloîtres médiévaux. Il rassemble également une importante collection de tapisseries. Il achète beaucoup d'objets qui ne sont pas destinés à quelque chose de précis et il lui arrive de revendre les œuvres qu'il a achetées. Malgré l'ampleur de sa collection, celle-ci est parfaitement gérée. Chaque œuvre ou groupe d'œuvres fait l'objet d'une fiche où sont répertoriés le nom du vendeur, la provenance, le prix payé, la destination de l'œuvre, éventuellement le nom de la personne à qui l'œuvre a été revendue et le prix de vente, le tout systématiquement accompagné d'une photographie ${ }^{51}$. Il ne développe pas un goût particulier pour l'art gothique mais en possède des quantités impressionnantes.

Quant à John D. Rockefeller Jr, esthète et philanthrope, il achète pour sa propre délectation mais aussi avec la volonté d'enrichir les collections des musées dont il est un grand bienfaiteur ${ }^{52}$. Il est également très engagé dans la restauration et la reconstruction de monuments en France, en particulier la cathédrale de Reims après les destructions de la Première Guerre mondiale.

\section{Vers une reconnaissance de l'art médiéval et une étude scientifique des œuvres}

17 L'art médiéval français reste encore malgré tout pour de nombreux amateurs un simple objet d'ornement, comme en témoigne l'article intitulé «Gothic Statuary as Decorations » qui paraît en $1920^{53}$. Illustré d'œuvres en vente chez Demotte, il ne contient aucune information scientifique sur les œuvres mais donne des conseils sur la meilleure manière de les présenter dans son intérieur. Dans le même temps, des départements consacrés à l'art médiéval vont commencer de se constituer dans les différents musées américains, avec à leur tête des conservateurs historiens de l'art. L'étude de cet art s'accompagne de la constitution de collections faisant l'objet d'achats réfléchis. C'est le cas du Fogg Art Museum à Harvard, où Arthur Kingsley Porter (1883-1933) $)^{54}$ achète en 1922 sur ses fonds propres ou fait acheter par le musée auprès de Demotte deux ensembles très importants de chapiteaux romans : douze chapiteaux venant de Moutiers-Saint-Jean et cinq des six chapiteaux venant de Saint-Pons-deThomières, présents chez Demotte, non plus pour leur valeur esthétique, patriotique ou spirituelle mais comme des œuvres d'art faisant l'objet d'études scientifiques ${ }^{55}$.

\section{NOTES}

1. Ces dernières années de nombreux ouvrages américains ont été consacrés à la constitution des collections d'art médiéval aux États-Unis et ce sujet a été abordé dans des expositions, comme à Newport en 2010: Gothic art in the gilded age: Medieval and Renaissance treasures in the GavetVanderbilt-Ringling collection [Sarasota, The John and Mable Ringling Museum of Art, 16 décembre 2009-4 avril 2010 ; Newport, The Preservation Society of Newport County, 8 mai-31 octobre 2010]. 2. Sur l'étude de ce fonds, voir Christine Vivet-Peclet, «Ce que révèle la documentation d'un grand marchand », dans Grande Galerie, novembre 2013, p. 18. 
3. Sur l'étude des antiquaires, voir Christine Vivet-Peclet, «Les sculptures du Louvre acquises auprès de Georges Joseph Demotte: de la polémique à la réhabilitation?", dans La revue des musées de France. Revue du Louvre, 3-2012, pp. 57-69.

4. Rémi Labrusse, «Paris, capitale des arts de l'Islam? Quelques aperçus sur la formation des collections françaises d'art islamique au tournant du siècle ", dans Bulletin de la Société de l'histoire de l'art français, 1997, pp. 283-285.

5. Louis Vauxcelles, «Les Arts - Exposition d'art gothique », dans Gil Blas, 13 septembre 1913, p. 4. 6. Barnard est à la fois sculpteur, collectionneur et marchand. Il étudie la sculpture à Chicago puis à Paris. Il reste en France pendant douze ans. Sur la personnalité de Barnard, voir par exemple : Timothy Husband, Creating the Cloisters, New York, Metropolitan Museum of Art, 2013. Les archives de George Grey Barnard sont conservées par plusieurs institutions dont les Archives of American Art à Washington D.C. qui rassemblent plusieurs de ces fonds sous forme d'originaux ou de microfilms lorsqu'ils viennent d'autres institutions.

7. Philadelphie, Philadelphia Museum of Art, Archives George Grey Barnard, correspondance Demotte, lettre du 15 septembre 1916.

8. Le carton du vernissage de l'ouverture de la galerie permet de connaître l'aspect extérieur de cette première boutique (carton retrouvé à : Archives of American Art, Washington D.C., The Carnegie Institute, Museum of Art Archives, box 41, folder 59).

9. Voir par exemple : «The art of old France », dans American Art News, 9 mars 1918, vol. 16, n 22, pp. 5-6.

10. Edmond Pottier, Le musée du Louvre pendant la guerre 1914-1918 - Notice lue à l'assemblée générale de la société des amis du Louvre le 5 février 1919, Paris, 1919.

11. André Michel, «Réouverture des salles de sculpture française au musée du Louvre ", dans Journal des débats politiques et littéraires, lundi 28 février 1916, p. 8.

12. L'Annonce aux Bergers (inv. RF 1607) et Deux hommes barbus, nimbés et couronnés (vieillards de l'Apocalypse ?) (inv. RF 1608, RF 1609), entrés au Louvre en 1914 et qui seront l'objet du scandale qui opposera Georges Joseph Demotte à l'un de ses employés Joanny Vigouroux en 1923. Voir sur cette affaire C. Vivet-Peclet, art. cit. note 3.

13. Par exemple dans Le Figaro, 2 mars 1916, p. 1.

14. Propos tenu dans le courrier déjà cité du 15 septembre 1916, voir note 6.

15. Famille de paysans dans un intérieur, inv. RF 2081.

16. Salle ouverte en 1870, portant le nom de Louis La Caze (1798-1869), qui donne au musée une collection de plus de cinq cents tableaux en 1869 (actuelle salle des bronzes antiques).

17. Maison alors en situation de monopole au musée pour la reproduction photographique et pour les éditions des catalogues du musée, illustrés ou non.

18. Séance du 27 février 1919 du comité consultatif des Musées nationaux, Archives nationales, 1 BB 38, p. 288.

19. Peintre et graveur, connu surtout comme créateur du théâtre d'ombres du Chat Noir.

20. Homme politique, académicien, plusieurs fois ministre et en particulier des Affaires étrangères en 1917, membre de la commission du Louvre, reçu à l'Académie française le 2 mai 1918 pour son action gouvernementale, il est tué le 9 octobre 1934 à Marseille, en même temps que le roi Alexandre de Yougoslavie, dans l'attentat perpétré par un terroriste croate.

21. Voir par exemple «Les enrichissements du Louvre depuis 1914 » dans Le Figaro, dimanche 28 novembre 1920, p. 1.

22. Voir note 14 .

23. Inv. RF 1663.

24. «L'actualité - Les livres d'art » dans L'art et les artistes, octobre 1920, pp. 129-130.

25. T. R., «Les accroissements des musées nationaux français. Le musée du Louvre depuis 1914 » dans La chronique des arts et de la curiosité, 31 décembre 1920, p. 178. 
26. François de Mély, «Le musée du Louvre - Ses accroissements de 1914 à 1920 ", dans Revue critique d'histoire et de littérature, $1^{\mathrm{er}}$ septembre 1921, pp. 323-326.

27. Musée du Louvre, Les accroissements des musées nationaux français - Le Musée du Louvre en 1920 Dons, legs et acquisitions (Tome III), Paris, Demotte, 1921.

28. Henri Rivière, Les dessins de Degas reproduits en fac-similé, Paris, Demotte, 1922.

29. Louis Réau, Étienne-Maurice Falconet, Paris, Demotte, 1922.

30. Georges Joseph Demotte, Salomon Reinach, La tapisserie gothique, Paris, Demotte, 1924.

31. Philadelphie, Philadelphia Museum of Art, Archives George Grey Barnard - correspondance Demotte, $1^{\text {er février } 1924 .}$

32. Bryn Athyn, Glencairn Museum, archives Raymond Pitcairn - courriers des 8 et 11 avril 1921.

33. Bulletin of the City Art Museum of St. Louis, vol. 7, n² 2, avril, 1922.

34. Sur ce musée, voir le catalogue de l'exposition qui s'est tenue en 2016 au musée du Louvre: Un musée révolutionnaire: le musée des Monuments français d'Alexandre Lenoir, Paris, éditions du musée du Louvre/Hazan, 2016.

35. Voir note 5.

36. Voir note 5 .

37. Sur les scandales qui ont émaillé l'activité de cette famille d'antiquaires, voir C. Vivet-Peclet, art. cit. note 2 .

38. Lois sur les Monuments historiques en 1913, sur la réglementation des exportations la même année, création de la Caisse des monuments historiques l'année suivante.

39. Il accompagne l'ouverture de ce musée de publicités dans la presse et de la publication d'un petit livret reprenant les articles les plus dithyrambiques qui ont accompagné l'inauguration. Barnard y expose ses motivations pour l'ouverture d'un tel lieu.

40. Cinquième enfant et seul fils de John Davison Rockefeller, fondateur de la compagnie américaine Standard Oil Company, il décide à partir de 1910 de se consacrer à la gestion de sa fortune et à ses actions de philanthropie.

41. Jean Vallery-Radot, «Pierres de France en Amérique», dans La Renaissance de l'art français et des industries de luxe, $\mathrm{n}^{\circ} 10,1926, \mathrm{pp}$. 533-546.

42. C'est la lecture de la correspondance entre Barnard et Demotte (archives conservées aux Archives of American Art, Washington D.C.) qui m'a mise sur la piste de ce projet, mais sans que le nom de Rockefeller ne soit mentionné. Mon intuition a été ensuite confirmée à la lecture de la correspondance entre Barnard, Rockefeller et l'architecte de ce dernier, Bosworth (archives conservées au Rockefeller Archive Center, Tarrytown).

43. Sur cet architecte, voir la thèse en cours d'Églantine Pasquier: Échanges culturels et architecturaux entre la France et les États-Unis, le rôle de l'architecte William Welles Bosworth (1869-1966), Paris, École du Louvre/Paris 1 Panthéon-Sorbonne.

44. Rockefeller Archive Center, office of the Messrs. Rockefeller records, Cultural Interests, Series E (FA314), Museums - George Gray Barnard, box 32, folder 328.

45. Photographies montrant ces œuvres sur la propriété : Rockefeller Archive Center, Rockefeller Family, albums, box 8, folder 1006, Pocanto Hills, Homes, Play House.

46. Article conservé dans un dossier documentaire du Metropolitan Museum of Art (référence incomplète).

47. Article signé Gregg Frederick James paru dans le New York Herald, dimanche [] octobre p. [] Article conservé dans un dossier documentaire du Metropolitan Museum of Art (référence incomplète).

48. Ensemble d'œuvres de Notre-Dame-de-la-Couldre à Parthenay du même ensemble que celles conservées au Louvre (voir note 11) : Entrée du Christ à Jérusalem (inv. S7n1), Deux hommes barbus, nimbés et couronnés (vieillards de l'Apocalypse?) (inv. S7n2).

49. Ed Gyllenhaal et Kirsten Hansen Gyllenhaal, The Bryn Athyn Historic District (Images of America), Charleston, Arcadia Publishing, 2011. 
50. E. Bruce Glenn, Glencairn, The Story of a Home, Bryn Athyn, Academy of the New Church, 1990. 51. Les archives de William Randolph Hearst concernant les acquisitions des œuvres d'art sont conservées à l'université de Long Island, Brookville (État de New York). Voir Catherine Larkin, The William Randolph Hearst Archive: An Emerging Opportunity for Digital Art Research and Scholarship, Long Island University, 2013

52. Voir note 39.

53. Peyton Boswell, « Gothic Statuary as Decorations », dans House \& Garden, vol. 37, $\mathrm{n}^{\circ}$ 6, 1920, pp. 44, 88, 90, 92.

54. Historien de l'art, médiéviste, spécialiste de l'architecture et de la sculpture romane, il enseigne à l'université de Harvard à partir des années 1920 mais également à la Sorbonne à Paris. 55. L'étude systématique de l'art médiéval français conservé dans les collections américaines a été engagée dans les années 1960 et se poursuit encore de nos jours avec tout d'abord la publication de Romanesque Sculpture in American Collections à partir de 1967 dans la revue Gesta (26 livraisons jusqu'en 1987, repris pour les vingt premiers dans deux volumes parus en 1979, t. I New England Museums, et 1999, t. II New York and New Jersey, Middle and South Atlantic States, the Midwest, Western and Pacific States), puis de Gothic Sculpture in American Collections publié de 1980 à 1984 dans Gesta puis en trois volumes publiés chez Brepols (1989, t. I, The New England Museums; 2001, t. II, The Museums of the Midwest ; 2016, t. III, The Museums of New York and Pennsylvania).

\section{RÉSUMÉS}

L'activité de l'antiquaire Georges-Joseph Demotte (1877-1923) va être dans la période qui précède la Première Guerre mondiale et celle qui lui succède un des moments cruciaux de la carrière de ce marchand. À Paris, tout d'abord, il décide de déménager vers les quartiers luxueux du $8^{\mathrm{e}}$ arrondissement et débute une nouvelle activité, celle d'éditeur d'art en publiant, en premier lieu, un ouvrage sur les accroissements du musée du Louvre pendant la guerre. À New York, il ouvre, quelques mois avant la fin de la guerre, une boutique en faisant le choix de se spécialiser dans l'art médiéval. L'arrivée de tels objets, dans le contexte particulier de l'immédiat aprèsguerre, va considérablement transformer le regard porté sur eux. Demotte va participer par ses ventes à l'enrichissement de collections américaines aussi diverses que celles d'Isabella Stewart Gardner (1840-1924), George Grey Barnard (1863-1938), Raymond Pitcairn (1885-1966), William Randolph Hearst (1863-1951) ou John D. Rockefeller Junior (1874-1960). Chacun de ces collectionneurs, aux profils et aux goût très variés, vont se référer dans les choix de présentation de leur collection à la France, à son patrimoine et à ses musées, mais moins au Louvre qu'à d'autres institutions comme le musée de Sculpture comparée ou le musée de Cluny.

The activity of the antiquarian Georges-Joseph Demotte (1877-1923) during the periods before and after the First World War was one of the crucial moments in his career. In Paris, he decided to move to the luxurious district of the eighth arrondissement and began a new activity, art publishing. The first book he published was on the growth of the Musée du Louvre during the war. A few months before the end of the war, he opened a boutique specialising in medieval art in New York. The arrival of such objects, in the particular context of the immediate post-war period, considerably transformed the way in which they were regarded. Through his sales, Demotte would contribute to the enrichment of American collections such as those of Isabella Stewart Gardner (1840-1924), George Grey Barnard (1863-1938), Raymond Pitcairn (1885-1966), 
William Randolph Hearst (1863-951) and John D. Rockefeller Junior (1874-1960). Each of these collectors, whose profiles and tastes were very different, referred in the way they chose to present their collections to France, its heritage and its museums, but less to the Louvre than to other institutions such as the Musée de Sculpture comparée and the Musée de Cluny.

\section{INDEX}

Keywords : medieval art, publishing, history of collections, history of taste, art market, American museums, Paris, musée du Louvre, First World War

Mots-clés : art médiéval, édition, histoire des collections, histoire du goût, marché de l'art, musées américains, Paris, musée du Louvre, Première Guerre mondiale

\section{AUTEUR}

\section{CHRISTINE VIVET-PECLET}

Après une formation à l'École du Louvre sur les ivoires médiévaux, en particulier sur les baisers de paix de la fin du Moyen Âge, Christine Vivet-Peclet effectue sa carrière professionnelle en gestion de l'information et de la documentation. D'abord en poste aux archives puis à la bibliothèque de l'École du Louvre, elle rejoint le département des Sculptures du musée du Louvre en 2004. En 2009 elle participe à l'exposition Les premiers retables au musée du Louvre à Paris et établit le corpus des œuvres des collections publiques publié en fin de catalogue. En 2014 elle collabore à l'exposition Saint Louis à la Conciergerie à Paris et en supervise la première partie consacrée à la représentation du souverain au cours du XIX ${ }^{\mathrm{e}}$ siècle. Elle est responsable d'un projet de recherche du musée du Louvre sur un fonds de plaques de verre photographiques reproduisant les œuvres passées chez une famille d'antiquaires au début du $\mathrm{xx}^{\mathrm{e}}$ siècle, les Demotte, qui est également l'objet de son doctorat.

***

After studying medieval ivories, particularly late medieval paxes, at the École du Louvre, Christine Vivet-Peclet pursued her career in information management and documentation. After working at the archives then at the library of the École du Louvre, she joined the Department of Sculptures at the Musée du Louvre in 2004. In 2009 she took part in the exhibition Les premiers retables at the Musée du Louvre in Paris and established the corpus of works from public collections published at the end of the catalogue. In 2014 she collaborated on the exhibition Saint Louis at the Conciergerie in Paris and supervised the first part of it, dedicated to the representation of the sovereign during the nineteenth century. She was responsible for a research project at the Musée du Louvre on a collection of photographic glass plates reproducing the works that passed through a family of antiquarians in the early twentieth century, the Demottes, which is also the subject of her $\mathrm{PhD}$ thesis. 